\title{
Supplement to: Remote sensing of ecosystem light use efficiency with MODIS-based PRI - the DOs and DON'Ts
}

\author{
A. Goerner ${ }^{\mathrm{a}, *}$, M. Reichstein $^{\mathrm{a}}$, E. Tomelleri ${ }^{\mathrm{a}}$, N. Hanan ${ }^{\mathrm{b}}$, \\ S. Rambal ${ }^{c}$, D. Papale ${ }^{d}$, D. Dragoni ${ }^{\text {e }}$, C. Schmullius ${ }^{\mathrm{f}}$ \\ ${ }^{a}$ Max Planck Institute for Biogeochemistry, 07745 Jena, Germany \\ ${ }^{\mathrm{b}}$ Natural Resources Ecology Lab, Colorado State University, USA \\ ${ }^{\mathrm{c}}$ CEFE-CNRS, 34293 Montpellier cedex 5, France \\ ${ }^{\mathrm{d}}$ Department of Forest Science and Environment, University of Tuscia, 01100 \\ Viterbo, Italy \\ ${ }^{\mathrm{e}}$ Department of Geography, Indiana University, Bloomington, Indiana, USA \\ ${ }^{\mathrm{f}}$ Friedrich-Schiller-University Jena, 07743 Jena, Germany
}
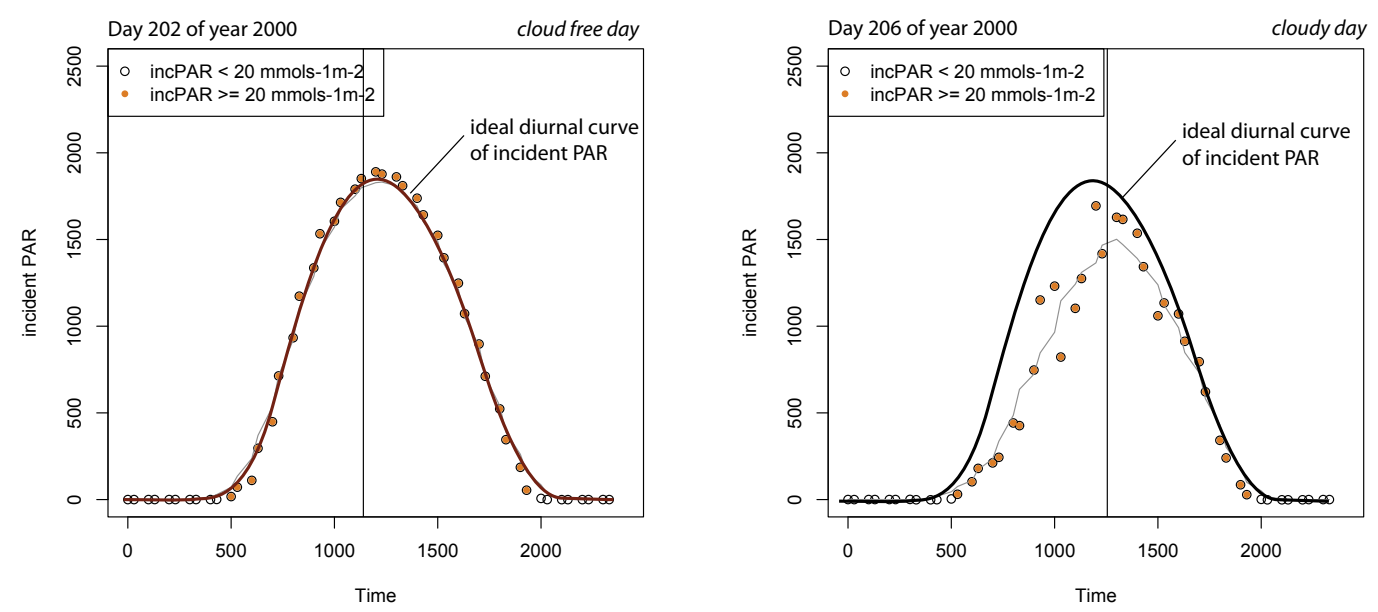

Fig. 1. Incoming photosynthetically active radiation at the FR-Pue site on a cloud free and a cloudy day, along with the curve that represents the diurnal course of PAR on a cloud free day for that particular site and time of year. Those curves were used to identify cloudfree days for all sites.

\footnotetext{
* Corresponding author

Email address: anna.goerner@bgc-jena.mpg.de (A. Goerner).

URL: http://www.bgc-jena.mpg.de/bgc-mdi/ (A. Goerner).
} 

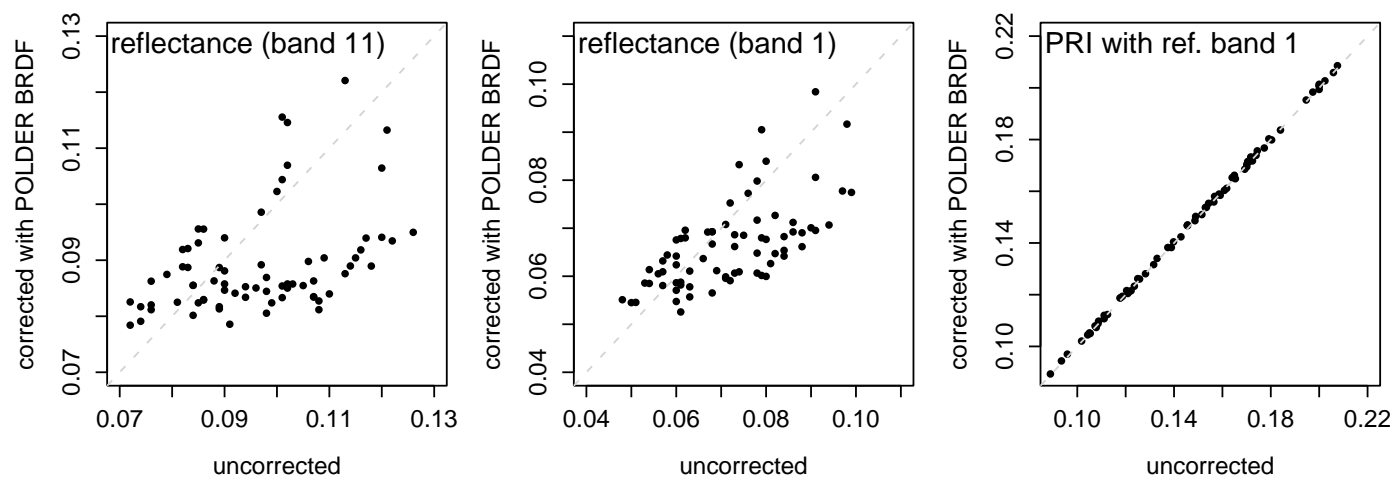

Fig. 2. Attempted correction for surface reflectance anisotropy with POLDER/ PARASOL BRDF parameters $(6 \times 6 \mathrm{~km})$ for 2002 at FR-Pue without explicit consideration of atmospheric effects. While the correction influences the reflectance of the individual bands that are used to compute PRI, these effects cancel each other out in the vegetation index itself.
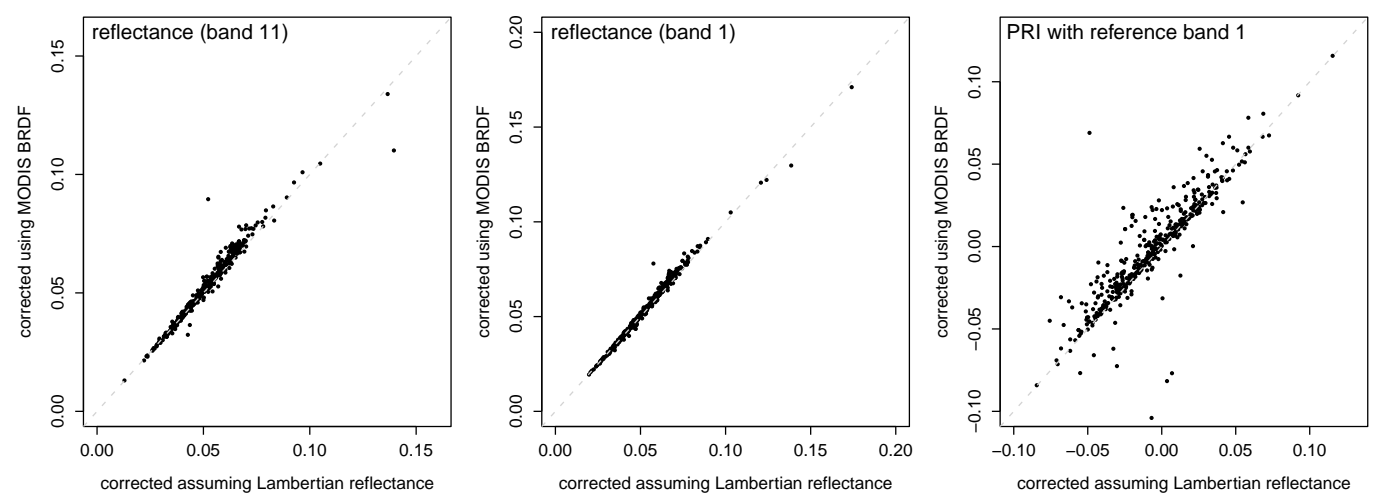

Fig. 3. Surface reflectance corrected for anisotropy with MODIS BRDF parameters versus surface reflection assuming an isotropic surface for 2000-2005 at FR-Pue. In both cases a correction for atmospheric effects has been perfomed using $6 \mathrm{~S}$ with the same input, so the difference is only due to applying the BRDF correction with $6 \mathrm{~S}$. The relatively small changes in reflectance $(2.0-3.5 \%)$ result in rather large differences in PRI (13-31\%). 


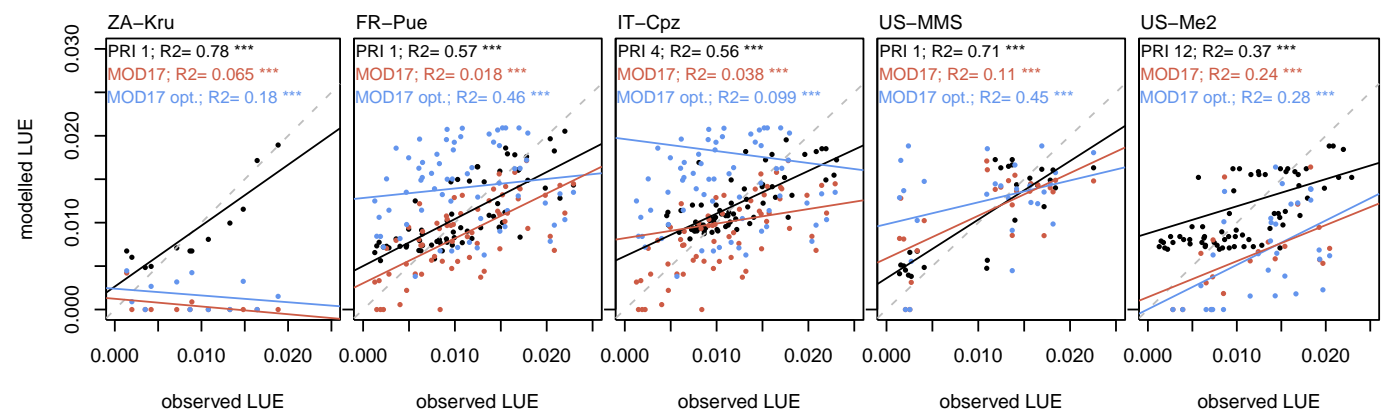

Fig. 4. LUE modelled from PRI (black), from MOD17 parameters (red), and from optimised MOD17 parameters (blue) versus ecosystem LUE calculated from fluxes and MODIS faPAR. Shown are only points for which a near-nadir PRI observation exists for the respective study site. Significance codes: $\mathrm{p}$ value $\leq 0.001$ : $_{\star \star \star} ; \mathrm{p}$

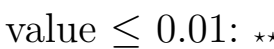




\begin{tabular}{|c|c|c|c|}
\hline 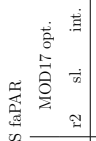 & 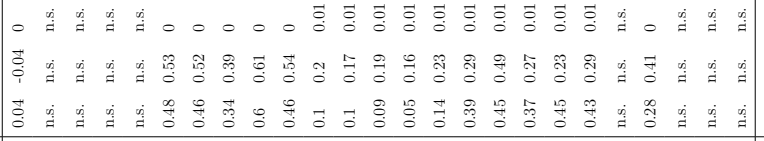 & & 营 \\
\hline 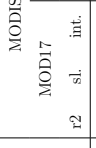 & 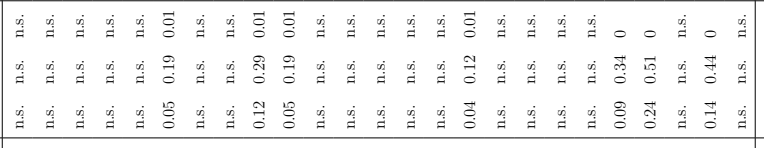 & & $\begin{array}{l}0 \\
0 \\
0 \\
0 \\
0 \\
0 \\
0 \\
0\end{array}$ \\
\hline 音 & 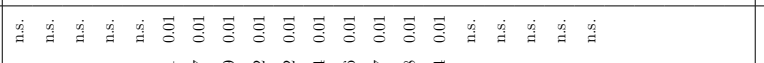 & 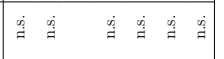 & \\
\hline 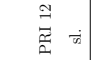 & 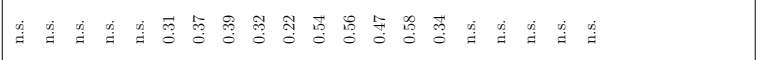 & 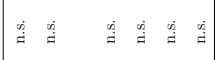 & $\underset{0}{\tau}$ \\
\hline & 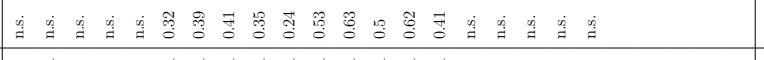 & 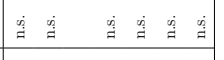 & $\stackrel{乛}{\varpi}$ \\
\hline & 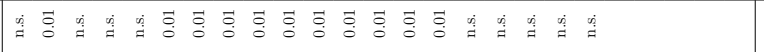 & 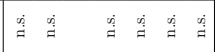 & $\underset{\Xi}{\overparen{J}}$ \\
\hline & 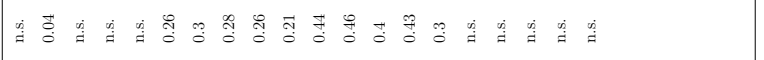 & 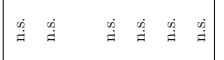 & \\
\hline & 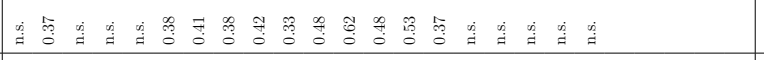 & $\stackrel{g}{g} \cong$ & 2 \\
\hline 音 & 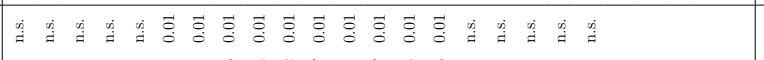 & 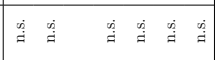 & $\stackrel{\Xi}{\rightleftarrows}$ \\
\hline 蓄缡 & 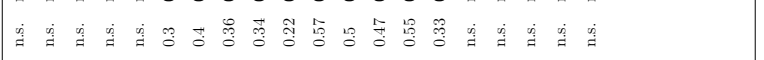 & 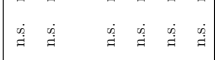 & \\
\hline & 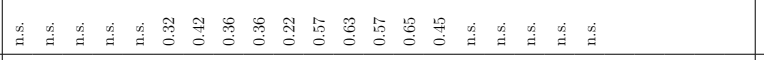 & $\cong \stackrel{g}{\cong} \cong$ & 4 \\
\hline 咅 & 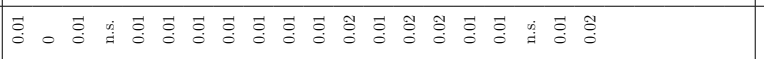 & 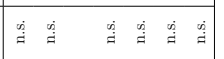 & \\
\hline $\overrightarrow{\tilde{z}} \overrightarrow{\bar{x}}$ & 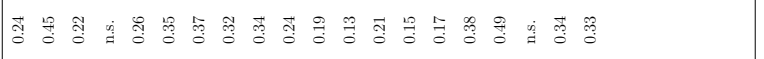 & 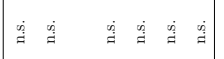 & \\
\hline$\approx$ & 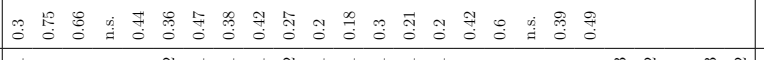 & 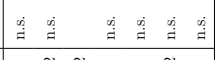 & \\
\hline 菃 & 参 & 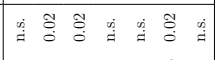 & \\
\hline$\vec{z} \frac{\pi}{x}$ & 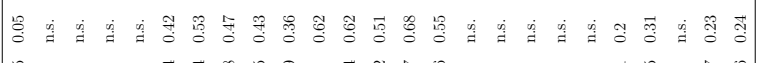 & 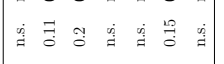 & \\
\hline & 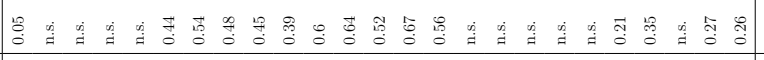 & 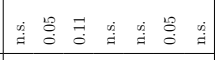 & 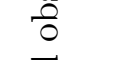 \\
\hline : & 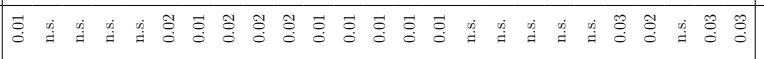 & 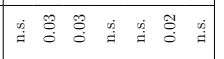 & $\vec{z}$ \\
\hline & 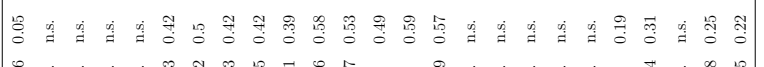 & 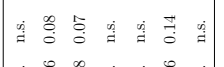 & $\begin{array}{ll}\pi \\
{[}\end{array}$ \\
\hline$\approx$ & 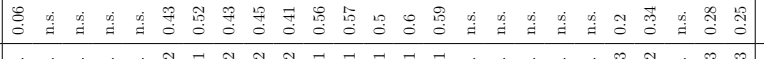 & 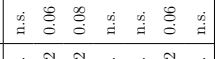 & 官 \\
\hline 音 & 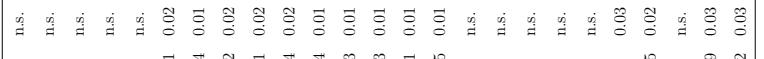 & 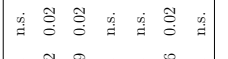 & $\overline{0}$ \\
\hline 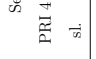 & 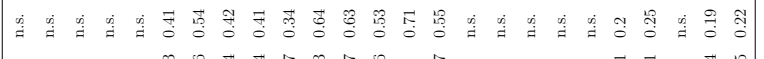 & 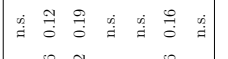 & $\stackrel{0}{\rightleftharpoons}$ \\
\hline$\approx$ & 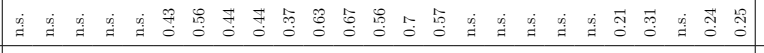 & 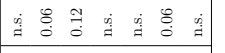 & to \\
\hline 荘 & 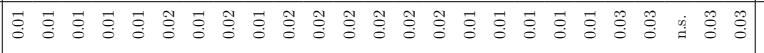 & 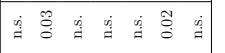 & $=$ \\
\hline $\overrightarrow{\tilde{z}} \overrightarrow{\bar{x}}$ & 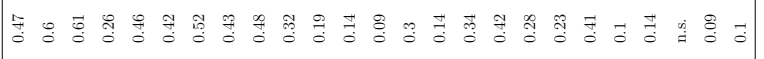 & 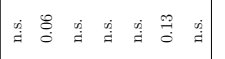 & 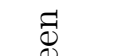 \\
\hline & 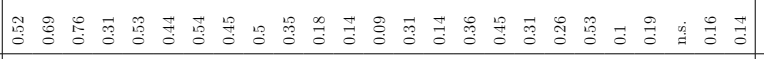 & 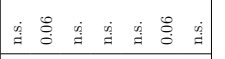 & \\
\hline 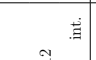 & 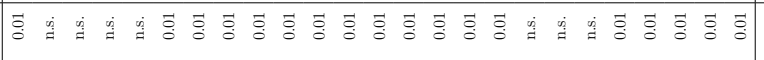 & $\vec{g}$ & .8 \\
\hline$\stackrel{\pi}{\pi}+\frac{1}{x}$ & 궁 & $\overbrace{i}^{\infty}$ & 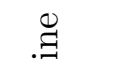 \\
\hline & 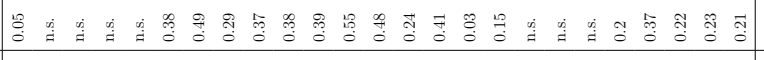 & 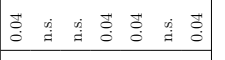 & \\
\hline 童 & 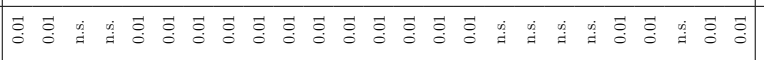 & 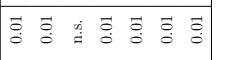 & \\
\hline${ }_{\tilde{z}}^{\circ} \rightarrow$ & 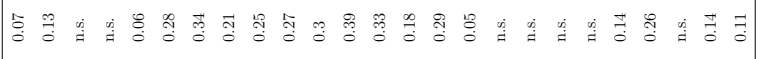 & 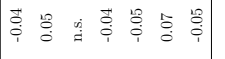 & \\
\hline & $\stackrel{8}{3}=$ & 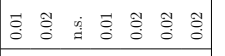 & \\
\hline$\dot{\Delta}$ & 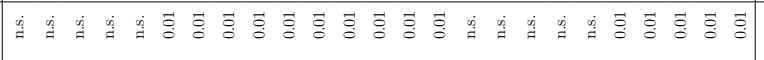 & 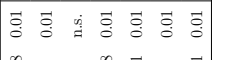 & \\
\hline & 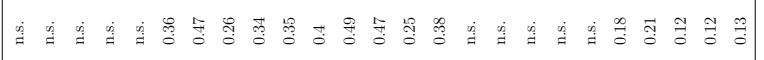 & 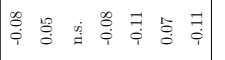 & \\
\hline & 영 & 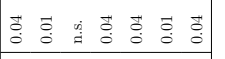 & \\
\hline 音 & 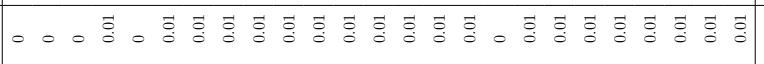 & 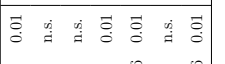 & \\
\hline $\begin{aligned} \vec{z} \\
\vec{x} \\
\Omega\end{aligned}$ & 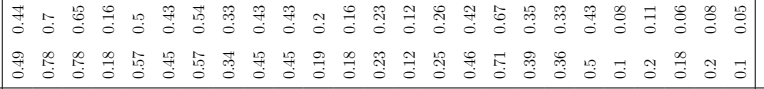 & 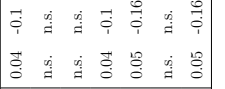 & $\stackrel{d}{\stackrel{d}{g}}$ \\
\hline 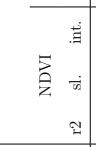 & 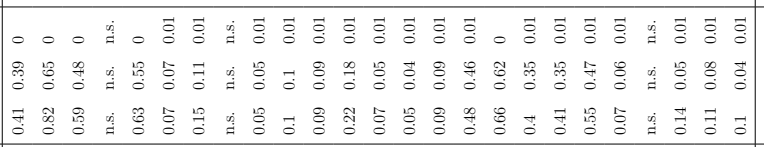 & & $\begin{array}{l}\vec{\sigma} \\
0\end{array}$ \\
\hline 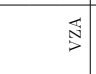 & 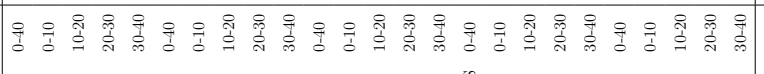 & & \\
\hline & 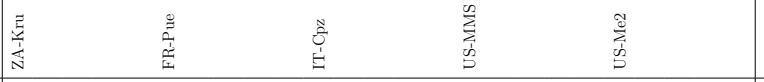 & 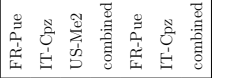 & \\
\hline$\Rightarrow 1$ & & & 7 \\
\hline
\end{tabular}

